\title{
$6-2014$
}

\section{Magnetotaxis as a Means for Nanofabrication}

\author{
Isaac Macwan
}

Zihe Zhao

Omar T. Sobh

Jinnque Rho

Ausif Mahmood

See next page for additional authors

Follow this and additional works at: https://digitalcommons.fairfield.edu/engineering-facultypubs Copyright 2014 World Scientific Publishing Company. All rights reserved.

The post-print version has been archived here with permission from the copyright holder. Electronic version of an article published in International Journal of High Speed Electronics and Systems, 23, 01n02, 2014,1450008. DOI: 10.1142/S0129156414500086 @ World Scientific Publishing Company. https://www.worldscientific.com/worldscinet/ijhses

\section{Peer Reviewed}

\section{Published Citation}

Macwan, Isaac, Zihe Zhao, Omar Sobh, Jinnque Rho, Ausif Mahmood, and Prabir Patra. "Magnetotaxis as a Means for Nanofabrication." International Journal of High Speed Electronics and Systems 23, no. 01 n02 (2014). https://doi.org/10.1142/S0129156414500086.

This item has been accepted for inclusion in DigitalCommons@Fairfield by an authorized administrator of DigitalCommons@Fairfield. It is brought to you by DigitalCommons@Fairfield with permission from the rightsholder(s) and is protected by copyright and/or related rights. You are free to use this item in any way that is permitted by the copyright and related rights legislation that applies to your use. For other uses, you need to obtain permission from the rights-holder(s) directly, unless additional rights are indicated by a Creative Commons license in the record and/or on the work itself. For more information, please contact digitalcommons@fairfield.edu. 


\section{Authors}

Isaac Macwan, Zihe Zhao, Omar T. Sobh, Jinnque Rho, Ausif Mahmood, and Prabir K. Patra 
International Journal of High Speed Electronics and Systems Vol. 23, No. 1 (2014) 1450008 (16 pages)

(C) World Scientific Publishing Company

DOI: 10.1142/S0129156414500086

\title{
Magnetotaxis as a Means for Nanofabrication
}

\author{
Isaac Macwan*
}

Department of Computer Science \& Engineering, University of Bridgeport, 221 University Avenue,

Bridgeport, CT 06604, United States

imacwan@my.bridgeport.edu

Zihe Zhao

Department of Biomedical Engineering,

University of Bridgeport, 221 University Avenue,

Bridgeport, CT 06604, United States

zihezhao@my.bridgeport.edu

\section{Omar Sobh}

Department of Biology,

University of Pennsylvania, 249 south 36th street,

Philadelphia, PA 19104, United States

osobh@sas.upenn.edu

Jinnque Rho

Department of Biology,

University of Bridgeport, 169 University Avenue,

Bridgeport, CT 06604, United States

rho@bridgeport.edu

\section{Ausif Mahmood}

Department of Computer Science and Engineering, University of Bridgeport, 221 University Avenue,

Bridgeport, CT 06604, United States

mahmood@bridgeport.edu

\section{Prabir Patra*}

Department of Mechanical and Biomedical Engineering,

University of Bridgeport, 221 University Avenue,

Bridgeport, CT 06604, United States

ppatra@bridgeport.edu

\begin{abstract}
Magnetotactic bacteria (MTB), discovered in early 1970s contain single-domain crystals of magnetite $\left(\mathrm{Fe}_{3} \mathrm{O}_{4}\right)$ called magnetosomes that tend to form a chain like structure from the proximal to the distal pole along the long axis of the cell. The ability of these bacteria to sense the magnetic field for displacement, also called magnetotaxis, arises from the magnetic dipole moment of this chain of magnetosomes. In aquatic habitats, these organisms sense the geomagnetic field and traverse the oxic-anoxic interface for optimal oxygen concentration along the field lines. Here we report an elegant use of MTB where magnetotaxis of Magnetospirillum magneticum (classified as AMB-1) could be utilized for controlled navigation over a semiconductor substrate for selective deposition. We examined $50 \mathrm{~mm}$ long coils made out of 18AWG and 20AWG copper conductors having
\end{abstract}


diameters of $5 \mathrm{~mm}, 10 \mathrm{~mm}$ and $20 \mathrm{~mm}$ for magnetic field intensity and heat generation. Based on the COMSOL simulations and experimental data, it is recognized that a compound semiconductor manufacturing technology involving bacterial carriers and carbon-based materials such as graphene and carbon nanotubes would be a desirable choice in the future.

Keywords: Magnetotactic bacteria; semiconductors; bioelectronics.

*Correspondence to Prabir Patra, ppatra@bridgeport.edu and/or Isaac Macwan, imacwan@my.bridgeport.edu

\section{Introduction}

According to the semiconductor industry roadmap maintained by ITRS (International Technology Roadmap for Semiconductors), the feature size of a single transistor on a silicon wafer has reached $22 \mathrm{~nm}$ in 2012. Test vehicles for the succeeding technology nodes such as MPUs (Microprocessor units), DRAMs (Dynamic Random Access Memories) and NAND-gate based flash drives are currently manufactured using a singleoptical exposure utilizing a double-patterning (DP) technique in order to extend the wavelength of exposure and to keep a constant numerical aperture (NA). ${ }^{1}$ As this cycle of scaling continues, the DP will be pushed even harder to accomplish the required shrinkage in the feature length, the potential solutions of which are being proposed with a condition that the necessary tools, masks and resists are in place. Also different nonlithographic or non-optical techniques are being explored to replace the silicon channel and source/drain regions with new, high mobility and high carrier velocity materials to sustain CMOS performance gains. With the advent of high-speed processing algorithms, microprocessor designers constantly strive for low power and faster transistor devices. One of the issues with this trend is that with the scaling down of the feature size (the length which the electrons must travel between the two junctions in order for the transistor device to conduct), the limit of the wavelength of EUV (Extreme Ultra-Violet) light is rapidly closing in. Furthermore, EUV immersion and double patterning lithography that started off from $193 \mathrm{~nm}$ and below requires a state-of-the-art digital fab (microelectronic fabrication facility) that costs greater than $\$ 5$ billion and 80 - 90 percent of this cost is in construction and capital equipment purchase and installation ${ }^{1}$. Considering all these factors, there is an urgent need for a new direction in the manufacturing of semiconductors, where restrictions on the wavelength of light can be eliminated through an altogether different approach compared to the conventional topdown strategies.

In nature, we have diverse species of organisms and it has been the tendency of humans to examine and mimic the phenomena and mechanisms that are available in the natural habitat. One such interesting organism is a bacteria called Magnetotactic bacteria (MTB), which was discovered in the early 1970s. ${ }^{2}$ These bacteria have in their cytoplasm single-domain crystals of magnetite $\left(\mathrm{Fe}_{3} \mathrm{O}_{4}\right)$ or greigite $\left(\mathrm{Fe}_{3} \mathrm{~S}_{4}\right)$ called magnetosomes that range from 35 to $120 \mathrm{~nm}$ in size. ${ }^{3}$ Such crystals tend to form a chain like structure from the proximal to the distal pole along the long axis of the cell and align to the bacterial 
cytoskeleton filament (made up of protein mamK $^{4}$ ) through an acidic protein mamJ. ${ }^{5}$ It is known that the ability of these bacteria to sense the magnetic field, also called magnetotaxis, ${ }^{6}$ arises from the magnetic dipole moment of this entire chain of magnetosomes acting as a large magnet. Such organisms being facultatively anaerobic are known to sense the geomagnetic field and traverse the oxic-anoxic interface in aquatic habitats for optimal oxygen concentrations. ${ }^{7}$ Furthermore, magnetotactic bacteria are known to be controlled by magnetic fields produced by either permanent magnets or coils of metal conductors producing magnetic flux around the current carrying conductor. ${ }^{8,9}$ A study on one of the species of MTB called MC-1 (Marine Coccus) involved displacement of polystyrene microbeads in a medium to detect pathogenic microorganisms. ${ }^{10}$ MTBs are also studied as controlled, MRI-trackable propulsion and steering systems for medical nanorobots in human vasculature. ${ }^{11}$

Here we come up with an alternative approach where magnetotaxis (sensitivity to the magnetic field) of a bacterial species is utilized for controlled navigation along a mesh of millimeter-sized solenoid coils and in turn for selective deposition on a substrate. The flagella bundles (FB) (about $12-20 \mathrm{~nm}$ in diameter) of MTB are able to produce a torque of approximately $4 \mathrm{pN}$ thereby displacing the cell giving it speeds ranging from 30 to $200 \mu \mathrm{m} / \mathrm{s}$ depending on the type of the species and the number of magnetosomes. ${ }^{8}$ Our preliminary results indicate that Magnetosprillum magneticum (classified as AMB-1) can be utilized for precise control and navigation of the potential semiconductor manufacturing nano-particles to achieve directed selective deposition. It is a nonlithographic technique that consists of two major interfaces, one between the bacterial entity and the inorganic molecule to be deposited and another between the inorganic molecule and the substrate (wafer). The former interface will guide the building material to the selected site over the substrate and the latter interface will aid the selective deposition. The purpose is to efficiently utilize the speed and size of these microorganisms to displace the molecules of semiconductor or compound semiconductor materials so as to selectively fabricate electronic components on a substrate. Our preliminary results indicate that such a compound technology involving bacterial carriers and carbon-based materials such as graphene and carbon nanotubes to fabricate information technology processing and memory units would be a possible alternative to the current EUV-based chip fabrication technology.

\section{Directed Self-Assembly as an Alternative Manufacturing Strategy}

The limit of the wavelength of EUV that is used in the present photolithography processes is about $15.4 \mathrm{~nm}$ below which there are tremendous challenges associated with the scaling of the components. Current chip manufacturing strategies can be divided into four major categories:

(1) Conventional methods, where efforts are made to improve the current equipments for better precision and resolution along with scaling of the MOSFETs such as doublepatterning, ${ }^{12,13}$ high-k dielectrics ${ }^{14}$ and strained silicon. ${ }^{15,16}$ 
(2) New materials, where replacement materials such as carbon-based materials graphene, carbon nanotubes, fullerenes and hydrogen saturated pentacene as well as hexabenzocoronene are used in place of conventional silicon-based materials. ${ }^{17}$ Other materials such as microbial nanowire networks, ${ }^{18}$ organic single-crystals, ${ }^{19}$ $\mathrm{DNA}^{20}$ and a combination of grapehene oxide, graphene, molybdenum disulphide $\left(\mathrm{MoS}_{2}\right)^{21}$, Strontium Titanate $\left(\mathrm{SrTiO}_{3}\right)$ and conventional materials such as silicon ${ }^{22}$ are also being investigated for replacing the current silicon-only based materials.

(3) Alternative methods, where new ways are explored for chip fabrication keeping the conventional working principles such as soft lithography, ${ }^{23}$ maskless lithography, ${ }^{24}$ molecular manipulation techniques, ${ }^{25,26}$ use of nanowires ${ }^{27,28}$ and tri-gate transistor technology. ${ }^{29}$

(4) Self-assembly, which can be further categorized into absolute (total) self-assembly ${ }^{30}$ and directed self-assembly. ${ }^{31,32}$

Recently there is a growing interest in an approach termed as a bottom-up strategy, where instead of etching the bigger substrate into smaller components, attempts are being made to use specially fabricated components such as carbon nanotubes or graphene in an assembly process to create nanostructures. However, the science and engineering of selfassembly can still be regarded in its infancy when it comes to the fabrication of a complex device such as a transistor on a silicon substrate. Currently, the size of the channel is about $22 \mathrm{~nm}$ and when compared to a molecule, it is still a significant number of atoms ( 92 atoms considering each silicon atom has a diameter of $0.24 \mathrm{~nm}$ ). Towards nanofabrication of semiconductors, self-assembly has been utilized for the fabrication of nanocrystals, nanowires and block copolymers. The techniques involved in the fabrication of such nanostructures can generate structures from few angstroms to microns in size. ${ }^{31}$ Some of the requirements that a self-assembly nanofabrication process below $50 \mathrm{~nm}$ must satisfy are production with nanometer precision, assembly at nanometer level, parallel nature, ability to structure in three dimensions and cost-effectiveness. The closest alternative to parallel lithography, which is cost-effective and can produce structures in three dimensions in a nanometer regime is soft lithography. However, the limitation of soft lithography is its serial nature that provides low throughput. Hence, self-assembly is the next best possible alternative considering the size of the present-day components on the wafer substrate. Some of the applications of self-assembly in the semiconductor industry are LED chip, silicon flash memory, MOS capacitor, electromechanical sensor and a chip along with an associated reader for DNA detection. ${ }^{32}$ Due to the limitations resulting from the influence of external forces and geometrical parameters, a new term was coined called "directed self-assembly", where the self-assembly is not treated as an automated perpetual process but rather dependent on a predefined control parameters. This is where simpler nanofabrication methods can be utilized to fabricate the components and various physical phenomena are utilized to assemble the fabricated components. In this regard, chemistry and fabrication go hand in hand and based on this convention, there are two classes of directed self-assembly processes: directed growth 
and directed assembly of the grown nanostructures. Some of the directed growth techniques that were demonstrated involved the use of nanowells for controlling the size and orientation of the nanocrystals, growth of nanowire arrays and the growth of nanopatterns such as $\mathrm{MoS}_{2} \cdot{ }^{33}$ Similarly, some of the physical phenomena that are used in the assembly of pre-fabricated components are fluidic based assembly - manipulation on the surfaces, ${ }^{34}$ electric and magnetic field mediated assembly ${ }^{35,36}$ and electrostatic assembly. ${ }^{37}$ Directed assembly of pre-fabricated nanostructures utilizing micro-organisms has been studied before for hydrophobicity, ${ }^{38}$ polystyrene microbead displacement, ${ }^{39}$ ordering $\mathrm{ZnS}$ nanocrystals ${ }^{40}$ as well as fabrication of metal, ${ }^{41}$ semiconductor and magnetic nanowires. ${ }^{42}$ Like any other micro-organisms, MTBs have also been utilized for collective tasks such as micro-robots in human arteriolocapillar networks for targeting tumor lesions, ${ }^{43}$ micro-component assembly, ${ }^{44}$ biosensor for pathogenic bacteria, ${ }^{45}$ carriers for polystyrene microbeads ${ }^{46}$ stacking of micro-cubes to form a structure similar to ancient pyramids ${ }^{47}$ and displacement of $100 \times 20 \times 15 \mu \mathrm{m}$ sized resin based microcomponents. ${ }^{48}$

\section{Methods}

AMB-1 (Magnetospirillum magneticum) (ATCC-700264) is a bipolar microaerophilic (facultatively anaerobic) micro-organism of the phylum $\alpha$-proteobacteria. This particular bacteria was selected due to their unique ability to grow both aerobically and anaerobically and their superior control through magnetotaxis due to the presence of intracellular nanoscale magnetite $\left(\mathrm{Fe}_{3} \mathrm{O}_{4}\right)$. The cultivation protocol as given by ATCC (American Type Culture Collection) for the revised MSGM (Magnetospirillum Growth Medium) was utilized both for the liquid as well as semisolid media preparation. The culture media per $1 \mathrm{~L}$ of distilled water included: $10 \mathrm{ml}$ Wolfe's vitamin solution, $5 \mathrm{ml}$ Wolfe's mineral solution, $2 \mathrm{ml} 0.01 \mathrm{M}$ ferric quinate, $0.45 \mathrm{ml} 0.1 \%$ resazurin, $0.68 \mathrm{~g}$ $\mathrm{KH}_{2} \mathrm{PO}_{4}, 0.12 \mathrm{~g} \mathrm{NaNO}$, 0.035g ascorbic acid, 0.37g tartaric acid, 0.37g succinic acid, $0.05 \mathrm{~g}$ sodium acetate and $1.3 \mathrm{~g}$ agar (for semi-solid media). After adding the chemicals, the media is checked for the $\mathrm{pH}$ of 6.75 and if needed, $0.5 \mathrm{M} \mathrm{NaoH}$ and HCL is used to set the $\mathrm{pH}$. The media is then autoclaved at $121^{\circ} \mathrm{C}$ for 15 minutes and about $1 \mathrm{ml}$ of inoculum is injected to a $10 \mathrm{ml}$ screw-cap test tube aseptically. The test tube is filled to the top leaving less than $1 \mathrm{ml}$ of headspace and is tightly screwed to have microaerophilic conditions. Finally, the culture is incubated at a temperature in the range of $26^{\circ}$ to $30^{\circ} \mathrm{C}$. Resazurin is added to the media to keep track of the dissolved oxygen. Initially the media with resazurin has a blue tinge of color but after a while it turns to pink and stays that way to indicate the presence of dissolved oxygen. If the conditions inside the test tube are close to anaerobic, then the blue tinge transforms to a colorless solution indicating that there is very little amount of dissolved oxygen in the media. 
Magnetotaxis of AMB-1 is investigated through locally generated magnetic field via a helical coil made up of copper conductor. It is well known that a line integral of $\mathbf{B}$ and $\mathrm{d} \mathbf{l}$ over a closed path is $\mu_{0}$ times the current enclosed by the path (Ampere's law):

$$
\oint B \bullet d l=\mu_{0} \mathrm{I}_{\text {enclosed }}
$$

The usefulness of the law is in determining the magnetic field around a long straight conductor as:

$$
\mathrm{B}=\left(\mu_{0} \mathrm{I}\right) /(2 \pi \mathrm{r})
$$

Effective directional control of the magnetic field via a coil instead of a straight conductor is emphasized as the field intensity is concentrated along the coil-axis and hence it is linear. It can be modeled as:

$$
\oint \vec{B} \cdot \overrightarrow{d l}=\mu_{0} \mathrm{~N} \mathrm{I}
$$

Similarly, the effect of heat on the community of AMB-1 is equally important to create favorable conditions even in the presence of heat generated by the coils. Short term effects of temperature on the abundance and diversity of magnetotactic cocci revealed the presence of four altogether different operational taxonomic units in one study, where it was found that $9^{\circ} \mathrm{C}, 15^{\circ} \mathrm{C}$ and $26^{\circ} \mathrm{C}$ temperature had relatively no effect on the MC-1 community and that above $37^{\circ} \mathrm{C}$, the number of MC-1 cells reduced drastically ${ }^{49}$. The temperature rise due to the current flowing through a conductor can be determined from the first principles using the mass (volume (Area x Length) and material density) of the conductor and the heat liberated (W) in the conductor can be determined using the action integral of the current and the resistivity $(\mathrm{R})$ of the conductor. Finally, the temperature rise $(\mathrm{T})$ can be determined by using the mass of the conductor and the specific heat as a result of injecting certain amount of heat $(\mathrm{W})$ :

$$
\mathrm{T}=\text { joules } \times 0.2389 \times \mathrm{W}
$$

It is known that the temperature co-efficient of resistivity is to be taken into account while calculating the final value of the temperature rise. The value obtained from equation (4) is to be reused to determine the changed value of the resistivity and based on this new value of resistivity, a new temperature rise is calculated. This cycle is repeated till the melting point of the material is reached. It is already known that the cultivation of such bacteria requires a temperature upto $30^{\circ} \mathrm{C}$. Thus, based on this information alone, the ability of the bacteria to survive beyond this temperature limit can be gauged.

The quantification of magnetic field and heat is carried out by COMSOL and MATLAB respectively.

Figure 1 shows the schematic of the proposed system along with the controller (FSM Finite State Machine). 


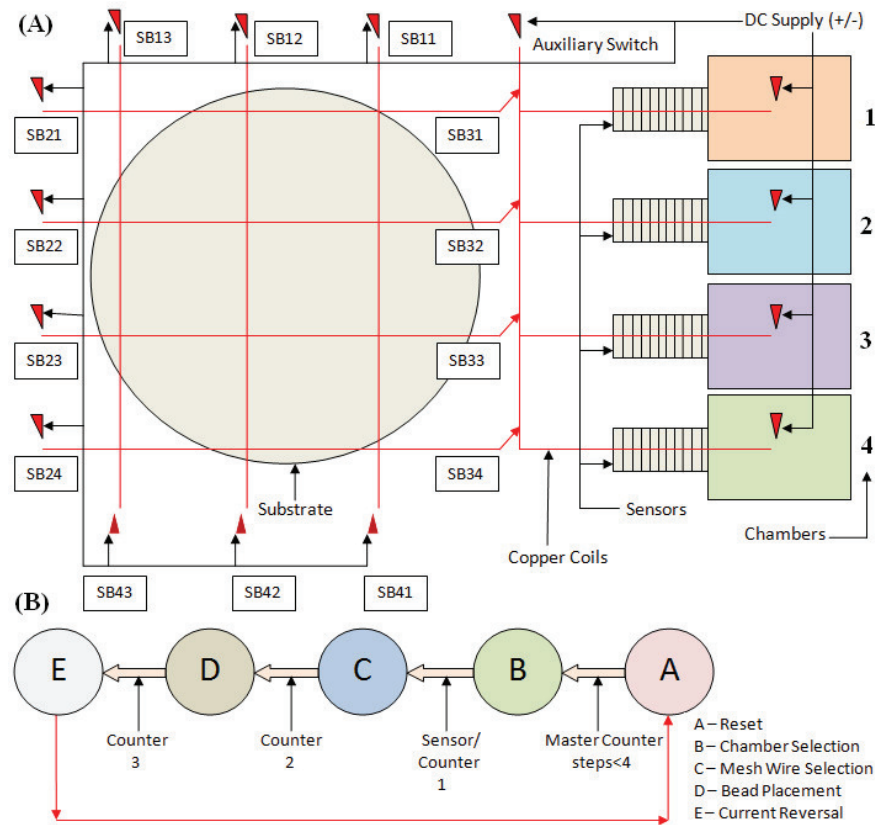

Fig. 1. Proposed scheme of the AMB-1 control system showing the basic micro-coil mesh structure (red colored conductors shown straight for clarity) along with the associated switches $\left(S_{x, y}\right)$ and chambers containing the physically-engineered (AMB-1 cells bound to nanoparticles) carriers (A) and a one-cycle FSM showing the five states of the controller involved in asserting the switches, thereby controlling the magnetic field (B).

As seen in Fig. 1(A), the four MEMS chambers contain carbon nanotube, graphene, metal oxide and copper nanoparticles. FSM (Finite State Machine) in Fig. 1(B) is an algorithm that is coded via VHDL (Very High Scale Integrated Circuit HDL) into the Altera DE-II cyclone 2 FPGA (field programmable gate arrays) chip, thereby acting as a controller. Other components of the system are micro-coils, sensor units, switches \& switchboards (SB). Figure 1 represents selective navigation of the bacterial species carrying said nanoparticles to a precise location on the wafer.

\section{Results and Discussion}

\subsection{AMB-1 Cultivation}

AMB-1 are cultured both through a semisolid petri dish technique as well using liquid media. The results are as shown in Fig. 2(A), (B) and (D). After the AMB-1 bacteria was inoculated at $30^{\circ} \mathrm{C}$ for 5 days, we could easily visualize that the medium in test tube 1 (control) was still clear but test tube 2 was turbid (Fig. 2(A)). Application of magnetic field via a small permanent magnet for 3 minutes revealed a visible dark spot near the permanent magent as shown in Fig. 2(B). Similarly, application of magnetic field to a petri dish showed displacement of AMB-1 cells to one side as shown in Fig. 2(D). 


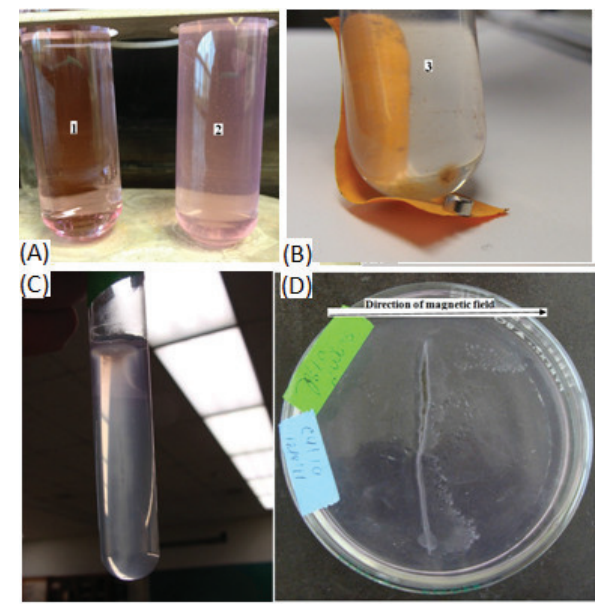

Fig. 2. (A) Test tube 1 (control) showing clear media indicating no contamination whereas test tube 2 shows turbidity indicating that the AMB-1 cells have cultured. Test tube 3 (B) shows a visible dark spot right over a permanent magnet indicating the magnetotaxis response of the AMB-1 cells. (C) AMB-1 cultivation in a semisolid media in a test tube showing visible tubidity right below the surface of the media confirming the microaerophilic nature of the AMB-1 cells. (D) AMB-1 cells cultured in a petri dish showing a visible vertical smear at the centre of the dish and cells migrating exclusively to the right side of the dish towards a magnetic pole confirming the magnetotaxis of the cells.

\subsection{Morphological observations of the AMB-1 cells}

Morphological observations of AMB-1 cells such as their shape and size and magnetosome characteristics are performed by optical as well as electron microscopy. The spiral shape of AMB-1 along with different sizes based on their growth phase using optical and scanning electron microscopy is as shown in Fig. 3(A) and 3(B). Figure 3(C) shows the gram-negative nature of the AMB-1 cells. TEM analysis revealed the morphological features of magnetosomes as shown in Fig. 4. High resolution image of the individual magnetosome show the crystals that are approximately $25-35 \mathrm{~nm}$ in size.

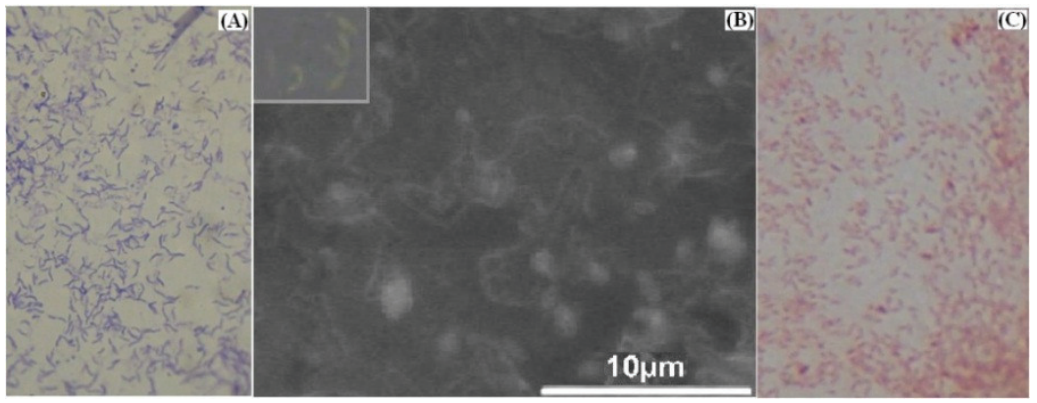

Fig. 3: (A) Simple staining of AMB-1 showing spiral morphology of the cells. Variations in the lengths are due to the different growth phases that the cells are going through. (B) SEM analysis of the AMB-1 cells clearly showing the spiral morphology. A comparison of the cell shapes with simple staining is done as indicated in the inset image. (C) Gram staining of the AMB-1 cells indicating gram-negative nature of the cells (red-colored) due to the application of counterstain called safranin. 


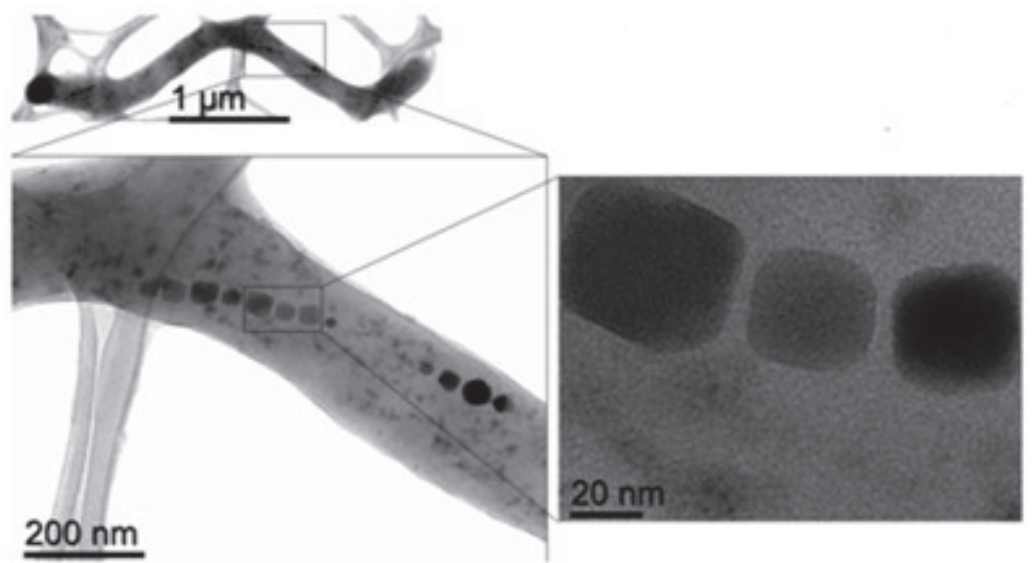

Fig. 4. TEM Analysis of Magnetospirillum magneticum (AMB-1) showing the size of individual cell along with intracellular magnetite crystals ( $\mathrm{Fe} 3 \mathrm{O} 4)$. The blown up views show the morphology and size of individual crystals.

\subsection{Motility Test}

Motility tests were performed using capillary race track technique (Fig. 5) and crossmicro slide technique (Fig. 6). AMB-1 strain consisting of motile cells, which are sensitive to magnetic field are separated and analyzed using optical microscope focused on the edge of the drop under the influence of externally applied magnetic field of $\sim 4 \mathrm{G}$.
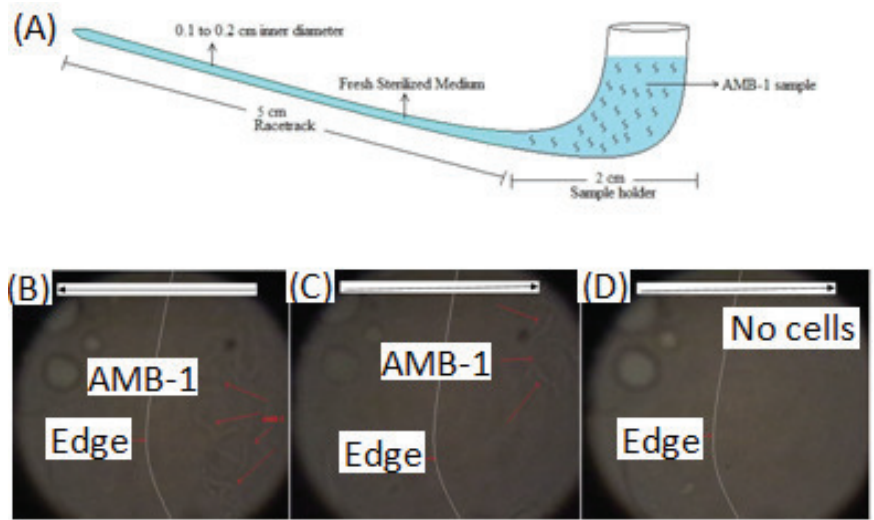

Fig. 5. Sample holder with AMB-1 cells attached to the capillary racetrack. Fig. 8. (A) AMB-1 cells accumulated to the edge of the drop towards the magnet. (B) AMB-1 cells moving away from the edge, following the direction of the magnetic field. (C) No AMB-1 cells in sight indicating an efficient magnetotaxis. 
In a cross micro-slide technique, four glass-slides are kept in a criss-cross configuration with a permanent magnet at the centre. After addition of the sample and keeping the cross slides configuration in the magnetic field at room temperature for $5 \mathrm{~min}$, the long track on each glass slide is cut into three pieces using sterilized cotton swab. From Fig. 6, the number of cells on the section 1 are the most, and the cellular density is reducing on sections 2 and 3 implying that $\mathrm{AMB}-1$ cells always move along the magnetic field lines.

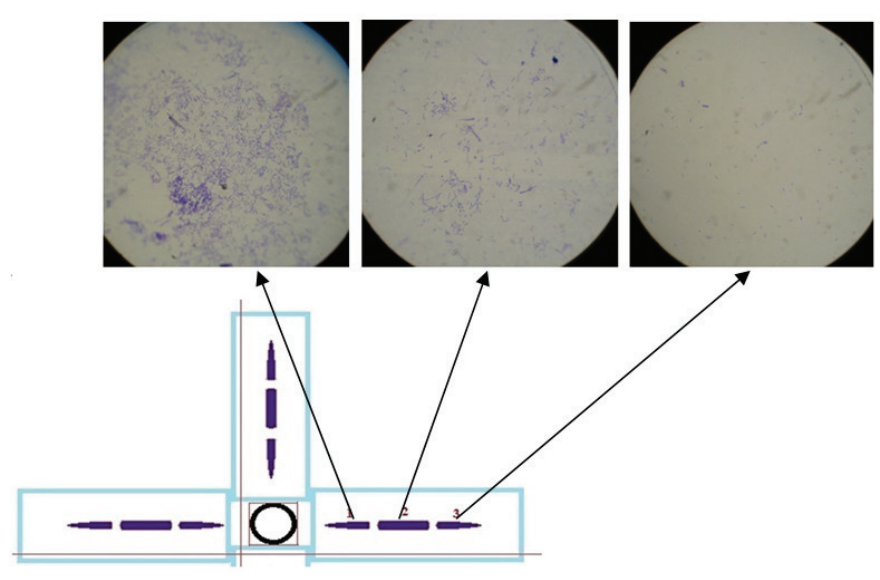

Fig. 6. Results of the CMS experiment indicating the increased cellular density near the magnet.

\subsection{Simulation results for copper coils using MATLAB and COMSOL}

MATLAB is used to model the relationship between the magnetic field intensity ' $\mathrm{B}$ ', current through the coil ' $\mathrm{I}$ ' and radial distance from the surface of the conductor ' $R$ ' as shown in Fig. 7. The 2-D curve shows what number of turns, $\mathrm{N}$ is needed for a $5 \mathrm{G}$ magnetic field for varying current and length of the coil. The 3-D curve on the other hand shows how ' $B$ ' will behave in the presence of both the variables ' $I$ ' and ' $R$ '. This complex relationship will govern not only the size and number of turns of the coil but also a limit to the radial distance beyond which 'B' would be difficult to control and hence the MTBs. 
Magnetotaxis as a Means for Nanofabrication
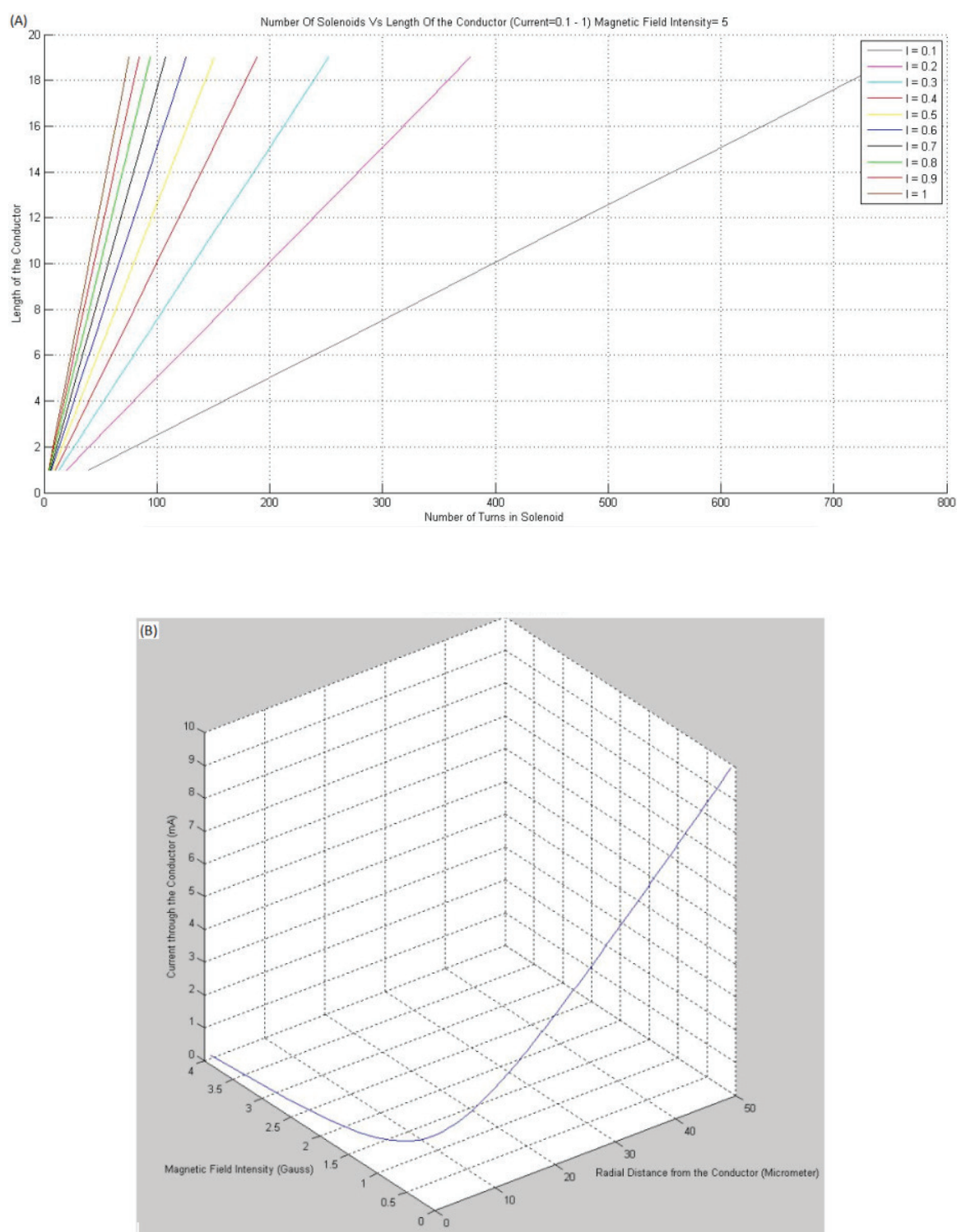

Fig. 7. (A) Length of coil VS Number of turns. (B) Magnetic field for a straight conductor. 
Similarly, FEA analysis is done using COMSOL's AC/DC module. The solenoid model for FEA analysis is created using 3D integrated CAD software from parametric called Pro-Engineer. The resulting simulation results for copper coil in bacterial media are as shown in Fig. 8.

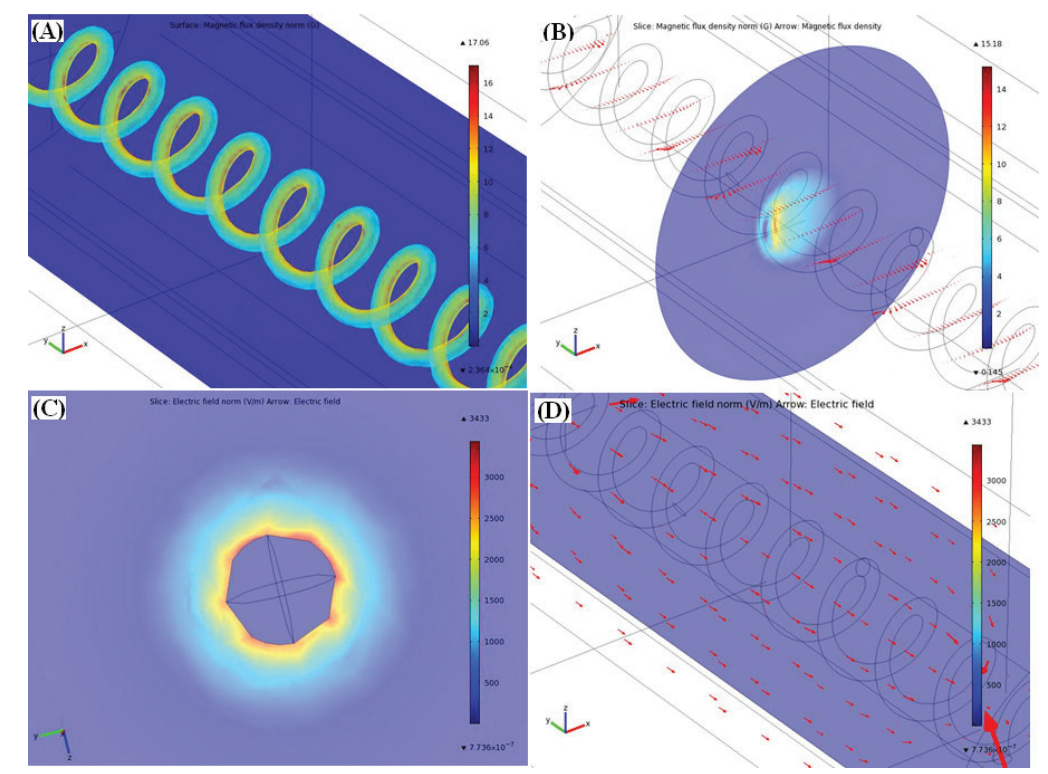

Fig. 8. (A), (B) Magnetic field intensity through a current carrying coil and its direction. (C), (D) Electric field intensity experienced by a magnetite crystal inside the coil and the direction of electric field from positive node to negative node.

Further, the magnetic field experienced by magnetite crystals inside the current carrying copper coil and the heat dissipation because of the current flowing through the coil is as shown in Fig. 9, which will govern the practical temperature limit beyond which AMB-1 cells will cease to travel and may lose their magneto-sensitive behavior. 

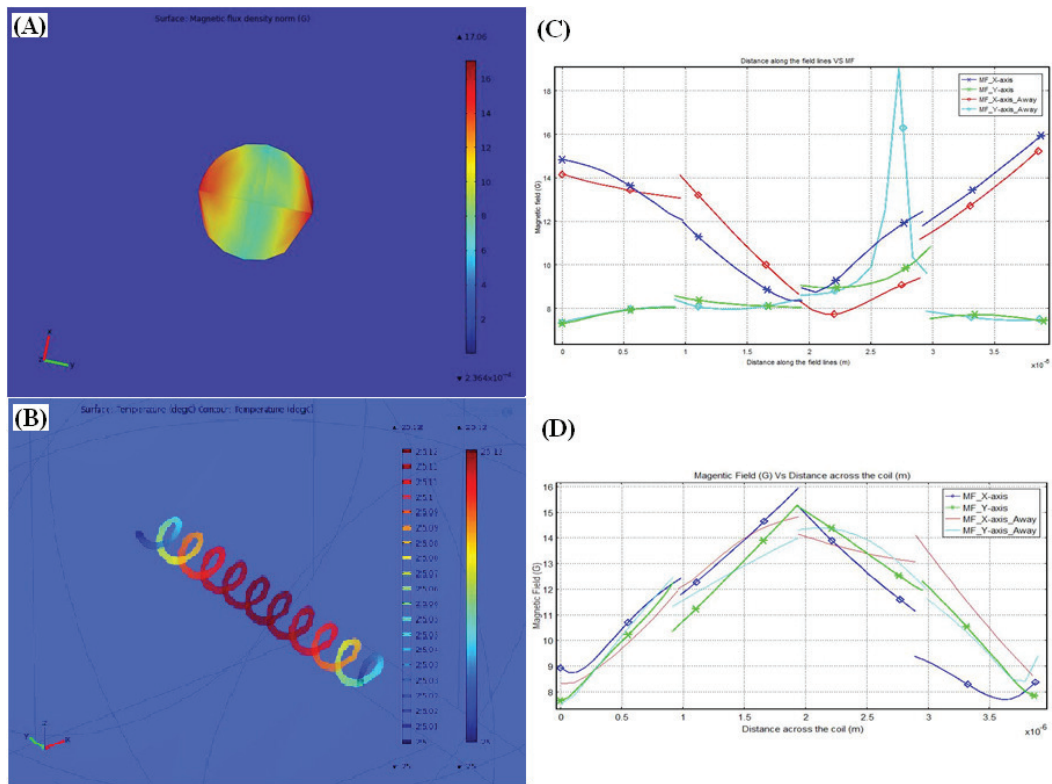

(D)

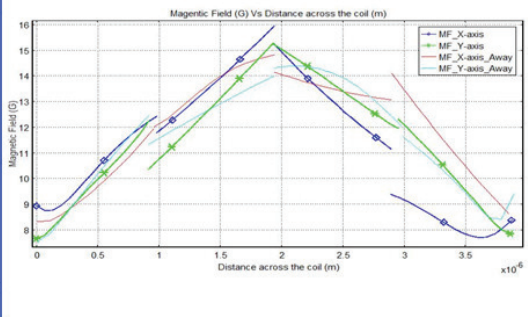

Fig. 9. (A) Magnetic field strength experienced by a magnetite crystal inside the copper coil. The magnetic field intensity taken along and across the magnetic field lines is as shown in (C) and (D). (B) shows a simulated heat dissipation for the copper coil and a corresponding temperature rise of $0.12^{\circ} \mathrm{C}$.

\section{Conclusion}

Results indicate that AMB-1 can be utilized for selective deposition tasks. Magnetotaxis of the individual AMB-1 cells can be controlled through a local magnetic field generated by solenoid coils. Simulation results from MATLAB and COMSOL on the generation of magnetic field and heat by solenoid coils give useful insights into the directional control of the bacterial entities. The components to be deposited such as carbon nanotubes and graphene are to be carried by the AMB-1 cells to the desired location on the substrate. These materials in turn could be functionalized on the AMB-1 flagellum through electrostatic/Van der waals interaction. Altera DE-II board acting as a controller would be an FPGA component to the selective deposition of the nanoparticles. Further investigation is necessary to compare the throughputs of conventional lithography and the bacterial operation. At present stage, it is the size of the selectively deposited particle that is of concern. However, in future using advanced algorithms, multiple AMB-1 cells could be used to manipulate multiple locations over the substrate and hence provide a parallel technique thereby multiplying the throughput. We envision a hybrid alternative technology to the current lithographic strategy where biological phenomena could be used to fabricate electronic circuits. 


\section{References}

1. Semiconductor Industry Association, International Technology Roadmap for Semiconductors (2011-2012); Future Fab International, Issue 39-40, October 2011-January 2012; available at http://public.itrs.net.

2. R. Blakemore, Science 190, 4212 (1975).

3. D. A. Bazylinski, D. Schuler, Microbe 4, 3 (2009).

4. Komeili, A., Z. Li, D. K. Newman, and G. J. Jensen, Science 311, 242-245 (2009).

5. Scheffel, A., M. Gruska, D. Faivre, A. Linaroudis, P. Graumann, J. M. Plitzko, and D. Schuler, Nature 440, 110-114 (2006).

6. Blakemore, R. P, Annu. Rev.Microbiol. 36, 217-238 (1982).

7. Bazylinski, D. A, ASM News 61, 337-343 (1995).

8. Martel S., Controlled bacterial micro-actuation, in Proc. Int. Conference on Microtechnologies in Medicine and Biology, Okinawa, Japan pp. 89, 92, 9-12 May 2006 doi: 10.1109/MMB.2006.251498.

9. Ryan C. Denomme, Zhao Lu, Sylvain Martel, A microsensor for the detection of a single pathogenic bacterium using magnetotactic bacteria-based bio-carriers: simulations and preliminary experiments", in Proc. of $29^{\text {th }}$ Ann. Int. conference of the IEEE Engineering Medicine Biology Society 'EMBS 2007', Lyon, France, pp. 99, 102 22-26 Aug. 2007 doi: 10.1109/IEMBS.2007.4352232.

10. Song H. P., Li X. G., Sun J. S., Xu S. M., Han X., Chemosphere 72, 616-621 (2008).

11. Martel S., Mohammadi M., Felfoul O., Lu Z., Pouponneau P., Int. J. Robot. Res. 28, 571-582 (2009).

12. C. Mack, Seeing Double, IEEE Spectrum, November 2008, [Online]. Available: http://spectrum.ieee.org/semiconductors/design/seeing-double

13. W. Shiu, H. Liu, J. Wu, T. Tseng, C. Liao, C. Liao, J. Liu, and T. Wang. Advanced selfaligned double patterning development for sub-30-nm DRAM manufacturing. In Proc. SPIE $7274,2009$.

14. G. D. Wilk, R. M. Wallace and J. M. Anthoy, J. Apl. Phy. 89, 10 (2001).

15. Thompson S.E. Armstrong M. Auth C. Cea S. Chau R. Glass G. Hoffman T. Klaus J. Zhiyong Ma, Mcintyre B. Murthy A. Obradovic B. Shifren L. Sivakumar S. Tyagi S. Ghani T. Mistry K. Bohr M. El-Mansy Y., IEEE Electron Device Letters 25, 4 pp. 191, 193 (2004).

16. M. V. Fischetti, F. Gamiz and W. Hansch, J. Apl. Phy. 92, 12 (2002).

17. M. Burghard, H. Klauk and K. Kern, Adv. mat. 21, 25-26 (2009).

18. Malvankar N. S., Vargas M., Nevin K. P., Franks A. E., Leang C., Kim B. C., Inoue K., Mester T., Covalla S. F., Johnson J. P., Rotello V. M., Tuominen M. T., Lovley D. R., Nature Nanotech. 6, 573-579 (2011).

19. Alejandro L. B., Stefan C. B. Mannsfeld M., M. Ling, S. Liu, R. J. Tseng, C. Reese, M. E. Roberts, Y. Yang, F. Wudl \& Z. Bao, Nature Lett. 444, 913-917 (2006).

20. Liu X., Aizen R., Freeman R., Yehezkeli O., Willner I., ACS Nano 6, 4 (2012).

21. Yu W. J., Li Z., Zhou H., Chen Y., Wang Y., Huang Y., Duan X., Nature Mat. 12, 246-252 (2013).

22. B. M. Kim, T. Brintlinger, E. Cobas, M. S. Fuhrer, Haimei Zheng, Z. Yu, R. Droopad, J. Ramdani, and K. Eisenbeiser, App. Phy. Lett. 84, 11 (2004).

23. Y. Xia, G. M. Whitsides, Ann. Rev. Mat. Sci. 28, 153-184 (1998). 
24. Pan L., Park Y., Xiong Y., Ulin-Avila E., Wang Y., Zeng L., Xiong S., Rho J., Sun C., Bogy D. B., Zhang X., Scientific Reports 1, 175 (2011).

25. J. H. Schon, H. Meng \& Z. Bao, Nature 413, 713-716 (2001).

26. S. I. Stupp, P. V. Braun, Science 277, 5330 (1997).

27. B. Weber, S. Mahapatra, H. Ryu, S. Lee, A. Fuhrer, T. C. G. Reusch, D. L. Thompson, W. C. T. Lee, G. Klimeck, L. C. L. Hollenberg, M. Y. Simmons, Science 335, 6064 (2012).

28. Cui Y., Duan X., Hu J., Lieber C. M., J. Phy. Chem. B 104, 22 (2000).

29. Doyle B., Boyanov B., Datta S., Doczy M., Hareland S., Jin B., Kavalieros J., Linton T., Rios R., Chau R., Tri-gate fully-depleted CMOS transistors: fabrication, design and layout, Symposium on VLSI Technology. Digest of Technical Papers. Kyoto, Japan, pp.133,134, 1012 June 2003. doi: 10.1109/VLSIT.2003.1221121.

30. E. C. P. Smits, Simon G. J. Mathijssen, Paul A. van Hal, Sepas Setayesh, Thomas C. T. Geuns, Kees A. H. A. Mutsaers, Eugenio Cantatore, Harry J. Wondergem, Oliver Werzer, Roland Resel, Martijn Kemerink, Stephan Kirchmeyer, Aziz M. Muzafarov, Sergei A. Ponomarenko, Bert de Boer, Paul W. M. Blom \& Dago M. de Leeuw, Nature 455, 956-959 (2008).

31. B. A. Parviz, D. Ryan, G. M. Whitesides, IEEE trans. Adv. Pack. 26, 3 (2003).

32. Wei L., A. M. Sastry, IEEE trans. Semiconductor Manufacturing 20, 4 (2007).

33. J. Henzie, J. E. Barton, C. L. Stender and T. W. Odom, Acc. Chem. Res. 39, 4 (2006).

34. F. Kim, S. Kwan, J. Akana and P. yang, J. Am. Chem. Soc. 123, 4360 (2011).

35. P. A. Smith, C. D. Nordquist, T. N. Jackson, T. S. Mayer, B. R. Martin, J. Mbindyo and T. E. Mallouk, Appl. Phys. Lett. 77, 1399 (2000).

36. H. Zhang, S. Boussaad, N. Ly and N. J. Tao, Appl. Phys. Lett. 84, 133 (2004).

37. Z. Tang, N. A. Kotov, M. Giersig, Science 297, 5579 (2002).

38. Mel Rosenberg, Appl. Envt. Microbiol. 42, 2 (1982).

39. D. B. Weibel, P. Garstecki, D. Ryan, W. R. DiLuzio, M. Mayer, J. E. Seto and G. M. Whitesides, PNAS 102, 34 (2005).

40. S. W. Lee, C. Mao, C. E. Flynn and A. M. Belcher, Science 296, 5569 (2002).

41. C. E. Flynn, S. W. Lee, B. R. Peelle and A. M. Belcher, Acta Materialia 51B, 19 (2003).

42. C. Mao, D. J. Solis, B. D. Reiss, S. T. Kottmann, R. Y. Sweeney, A. Hayhurst, G. Georgiou, B. Iverson, A. M. Belcher, Science 303, 5655 (2004).

43. S. Martel, Towards MRI-controlled ferromagnetic and MC-1 magnetotactic bacterial carriers for targeted therapies in arteriolocapillar networks stimulated by tumoral angiogenesis, in Proc. 28th Ann. Int. conference of the IEEE Engineering Medicine Biology Society 'EMBS 06', New York, NY, 30 Aug. - 03 Sept., 2006.

44. S. Martel, W. Andre, M. Mahmood, Z. Lu, O. Felfoul, Towards swarms of communicationenabled and intelligent sensotaxis-based bacterial micro-robots capable of collective tasks in an aqueous medium, in Proc. of the 2009 IEEE Int. Conf. on Robotics and Automation 'ICRA 09', Kobe, 12-17 May 2009.

45. Martel S., Magnetotactic phage-based microrobotic systems for the detection of live bacteria, in Proc. of The First IEEE/RAS-EMBS Int. Conf. on Biomedical Robotics and Biomechatronics 'BioRob 2006', Pisa, 20-22 Feb. 2006.

46. S. Martel, C. C. Trembley, S. Ngakeng, G. Langlois, App. Phy. Lett. 89, 23 (2006). 


\section{Macwan et al.}

47. S. Martel \& M. Mohammadi, Using a swarm of self-propelled natural microrobots in the form of flagellated bacteria to perform complex micro-assembly tasks, in Proc. of 2010 IEEE Int. conf. on Robotics and Automation 'ICRA', Anchorage, Alaska, 3-7 May 2010.

48. Khoshbakht M. E., Mokrani N., Mohammadi M., Martel S., Impact of the geometrical features of micro-components in bacterial micro-assemblies. in Proc. of 5th Int. conf. on micromanufacturing 'ICOMM/4M 2010', Madison, Wisconsin, 5-8 Apr. 2010.

49. Lin W., Wang Y., Pan Y., MicrobiologyOpen 1, 1 (2012). 